\title{
Jovian Electrons In The Inner Heliosphere: A Parameter Study On Intensity Profiles Near Earth
}

\author{
Adrian Vogt* \\ Christian-Albrechts-Universität zu Kiel \\ E-mail: vogtephysik.uni-kiel.de \\ Phillip Dunzlaff \\ Christian-Albrechts-Universität zu Kiel

\section{Bernd Heber} \\ Christian-Albrechts-Universität zu Kiel \\ E-mail: heberephysik. uni-kiel.de

\section{Andreas Kopp} \\ Christian-Albrechts-Universität zu Kiel \\ E-mail: kopp@physik.uni-kiel.de

\section{Patrick Kühl} \\ Christian-Albrechts-Universität zu Kiel \\ E-mail: kuehl@physik.uni-kiel.de
}

\section{Roelf Du Toit Strauss}

North-West University, Potchefstroom Campus

E-mail: dutoit.straussenwu.ac.za

\begin{abstract}
Although the main processes are well known, the transport parameters of charged particles in the inner heliosphere are still under investigation. A newly developed GPU-accelerated code to solve Parker's transport equation by means of stochastic differential equations (SDEs) offers the possibility to perform extensive parameter studies. Investigating electrons originating from the Jovian magnetosphere, we will present the potential of the code as well as first results of the time profile near Earth as a function of varying the solar wind speed, the strength of the diffusion and relation between the parallel and the perpendicular diffusion coefficient.
\end{abstract}

The 34th International Cosmic Ray Conference,

30 July- 6 August, 2015

The Hague, The Netherlands

\footnotetext{
* Speaker.
} 


\section{Introduction}

Recent developements made high performance calculations on graphic processing units (GPUs) accessible. Since the introduction of Nvidia's Compute Unified Device Architecture (CUDA) the computation power of GPUs has been applied on problems in various scientific fields. The massively parallel design of GPUs leads to an increase of the run time performance of 10 up to 60 [Dunzlaff et al.(2015)] for problems which can be parallelized as, in this case, the solution of the stochastic differential equation (SDE) formulation of Parkers transport equation.

Despite of the sufficient knowledge of the main processes, the values of the transport parameters are still under debate. Here, electrons originating from the Jovian magnetosphere are used to investigate the solar wind speed $u_{S W}$ and the diffusion tensor $\hat{\kappa}$, including the ratio $\chi=\kappa_{\perp} / \kappa_{\|}$ between the perpendicular and parallel diffusion coefficients.

With the code presented in the following we are given the opportunity to achieve a deeper and more quantitative understanding of charged particle transport during solar quiet time conditions in order to evaluate parameter sets for future modelling approaches.

\section{The modelling approach}

The transport of charged particles in the heliosphere can be described by the Parker equation [Parker (1965)] as the evolution of a time dependent distribution function $f$. The actual differential particle intensity $j$ is linked to $f$ via the particle rigidity $P=\sqrt{E\left(E+2 E_{0}\right)}$ as

$$
j=P^{2} f .
$$

with $E_{0}$ as the rest energy and $E$ as the kinetic energy of the particle. Parker's transport equation (TPE) contains the relevant processes in the supersonic solar wind in four terms

$$
\frac{\partial f}{\partial t}=\underbrace{S}_{\text {Source }}+\underbrace{\vec{\nabla} \cdot(\hat{\kappa} \cdot \vec{\nabla} f)}_{\text {Diffusion }}-\underbrace{\vec{u}_{S W} \cdot \vec{\nabla} f}_{\text {Convection }}+\underbrace{\frac{1}{3}\left(\vec{\nabla} \cdot \vec{u}_{S W}\right) \frac{\partial f}{\partial \ln E}}_{\text {Adiabatic Energy Changes }},
$$

Thereby the solar wind flow $\vec{u}_{S W}$ is assumed to be constant and directed radially outward:

$$
\vec{u}_{S W}=v_{S W} \cdot \vec{e}_{r},
$$

and the symmetric diffusion tensor $\hat{\kappa}$ is defined according to [Burger et al.(2008)] in coordinates aligned to the heliospheric magnetic field lines and transformed into the sun-centered spherical coordinate system

$\hat{\kappa}=\left(\begin{array}{ccc}\kappa_{\|} & 0 & 0 \\ 0 & \kappa_{\perp r} & 0 \\ 0 & 0 & \kappa_{\perp \theta}\end{array}\right) \quad \Longrightarrow \quad \hat{\kappa}_{\text {sphere }}=\left(\begin{array}{ccc}\kappa_{\|} \cos ^{2} \psi+\kappa_{\perp} \sin ^{2} \psi & 0 & \sin \psi \cos \psi\left(\kappa_{\perp}-\kappa_{\|}\right) \\ 0 & \kappa_{\perp} & 0 \\ \sin \psi \cos \psi\left(\kappa_{\perp}-\kappa_{\|}\right) & 0 & \kappa_{\|} \sin ^{2} \psi+\kappa_{\perp} \cos ^{2} \psi\end{array}\right)$

with the Parker angle $\psi$ between the field lines of the nominal Parker field and the radial direction from the Sun, thus taking into account the geometric structure of the heliospheric magnetic field. 
The parallel diffusion coefficient $\kappa_{\|}$within the diffusion tensor is considered as

$$
\kappa_{\|}(r)=\frac{v \lambda_{\|}(r)}{3}
$$

with the particle speed and the mean free path $\lambda_{\|}(r)$, normalized to the value $\lambda_{0}$ at $r_{0}=1$ AU via

$$
\lambda_{\|}(r)=\frac{\lambda_{0}}{2}\left(1+\frac{r}{r_{0}}\right) .
$$

The relation between the parallel and the perpendicular diffusion coefficients is assumed to be linear

$$
\kappa_{\perp r / \theta}(r)=\chi \kappa_{\|}(r) .
$$

while the two perpendicular components $\kappa_{\perp r}$ and $\kappa_{\perp \theta}$ are treated as equal.

\subsection{Stochastic Differential Equations (SDEs)}

Several methods to solve the TPE have been applied to model the transport of CRs in the heliosphere. Because analytical solutions are available only for a few and limited cases a numerically approach is inevitable. The most commonly used numerical modulation models are based on finitedifference numerical schemes. However, over the last decade the focus has shifted to alternative algorithms, such as the method of stochastic differential equations (SDEs), which is numerically stable and independent of grid size limitations. Furthermore SDEs can be solved both forward and backward in time, depending on the scientific goal.

The SDE method as described in [Zhang (1999)] and [Kopp et al.(2012)] in detail, has already been successfully applied to heliospherical objectives by [Strauss et al.(2013)]. In this method the corresponding Langevin equation

$$
d \underline{r}=\underline{A} d t+\mathfrak{B} \cdot d \underline{\omega}
$$

is solved. In this generalized form with $\underline{r}=(\vec{r}, E)$ the convective terms $(\underline{A})$ are taken into account via the time-step $d t$ whereas the diffusive terms $\left(\mathfrak{B}^{T} \mathfrak{B}=2 \hat{\kappa}\right)$ contribute via the Wiener process $d \underline{\omega}=\underline{\eta} \sqrt{d t}$ with the vector $\underline{\eta}$ of Gaussian distributed random numbers, leading to a random walk through the phase space. For the 3D time dependent TPE as it is investigated in this study, the equivalent SDEs are, according to [Strauss et al.(2011a)]:

$$
\begin{aligned}
& d r= {\left[\frac{1}{r^{2}} \frac{\partial}{\partial r}\left(r^{2} \kappa_{r r}\right)+\frac{1}{r \sin \theta} \frac{\partial \kappa_{r \phi}}{\partial \phi}-u_{S W}\right] d s } \\
&+\sqrt{2 \kappa_{r r}-\frac{2 \kappa_{r \phi}^{2}}{\kappa_{\phi \phi}}} d \omega_{r}+\frac{\sqrt{2 \kappa_{r \phi}}}{\kappa_{\phi \phi}} d \omega_{\phi} \\
& d \theta= {\left[\frac{1}{r^{2} \sin \theta} \frac{\partial}{\partial \theta}\left(\sin \theta \kappa_{\theta \theta}\right)\right] d s+\frac{\sqrt{2 \kappa_{\theta \theta}}}{r} d \omega_{\theta} } \\
& d \phi= {\left[\frac{1}{r^{2} \sin ^{2} \theta} \frac{\partial \kappa_{\phi \phi}}{\partial \phi}+\frac{1}{r^{2} \sin \theta} \frac{\partial}{\partial r}\left(r \kappa_{r \phi}\right)\right] d s+\frac{\sqrt{2 \kappa_{\phi \phi}}}{r \sin \theta} d \omega_{\phi} } \\
& d E= {\left[\frac{1}{3 r^{2}} \frac{\partial}{\partial r}\left(r^{2} u_{S W}\right) \Gamma E\right] d s } \\
& \quad \text { with } \quad \Gamma=\frac{E+2 E_{0}}{E+E_{0}}
\end{aligned}
$$




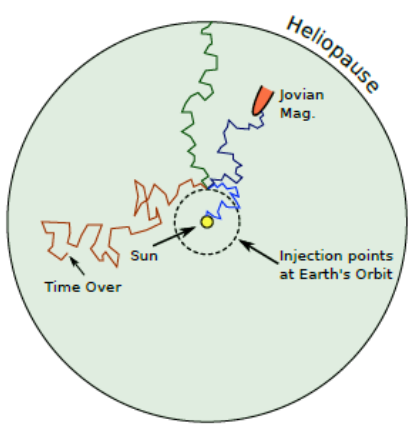

Figure 1: Schematic sketch of the simulation setup in the ecliptic plane. For a sample injection point at the Earth orbit, the four different boundaries are shown: the Sun (blue trajectory), the Jovian magnetosphere (marine), the Heliopause (green) and the temporal boundary, when the maximum backward time is reached (red).

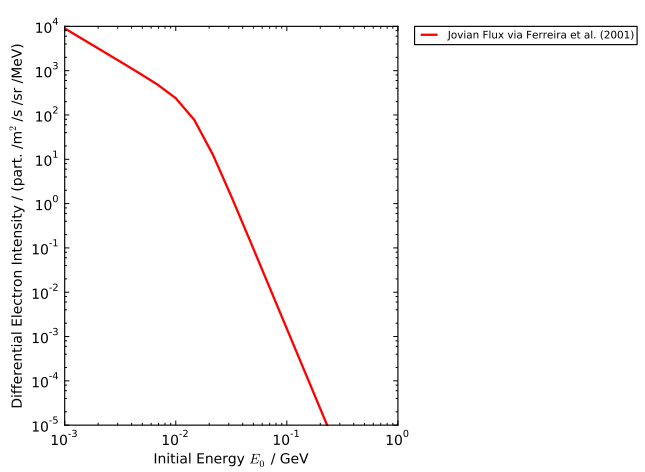

Figure 2: The Jovian electron source spectrum by [Ferreira et al.(2001)] used to weight the trajectories which reached the corresponding spatial boundaries of the phase space.

describing the adiabatic energy changes. The SDEs are implemented in their time-backward notation, with $s$ as the backward time and the incrementing backward timestep $d s$ instead of the forward timestep $d t$ used in Eqn. 2.7 .

\subsection{The General Setup}

The SDEs are solved using the Euler-Maruyama scheme. Because of better statistics, the timebackward approach is adopted for the simulation setup. In this approach, the differential intensity $j$ is obtained at the initial phase space point $\left(r^{0}, E^{0}\right)$, which could be also called the "observational point", at the backwards time $s=0$.

Via the backward timestep $d s$ the evolution of the initial phase space point for $s>0$, is calculated iteratively until a spatial $\left(r^{\text {exit }}\right)$ or temporal $\left(s^{\text {exit }}\right)$ boundary is reached. As illustrated in Figure 1 three spatial boundaries are possible in the simulation setup. Besides the Sun, there is the Jovian magnetosphere and the heliopause at $R_{H P}$. Due to the backward integration in time, the pseudo-particle does gains instead of losing energy while performing its random walk from the "observational point" to its "source". Thus, the injection energy $E^{0}$ can be interpreted as the particle energy when it hits a detector at the injection point, whereas the exit energy $E^{\text {exit }}$ is the energy the particle left its source with, for instance the Jovian magnetosphere.

To obtain $j\left(E^{0}, r^{0}\right)$ requires good statistics and, thus, a large number of pseudo-particle trajectories. Figure 2 shows the Jovian source spectrum by $f_{J o v}(E)$ [Ferreira et al.(2001)] which is used to weight the phase space trajectories, according to the source spectrum $E^{\text {exit }}$ via

$$
j\left(r^{0}, E^{0}\right)=\frac{P_{0}^{2}}{N} \sum_{k=1}^{N} f_{J o v}\left(E_{k}^{e x i t}\right)
$$




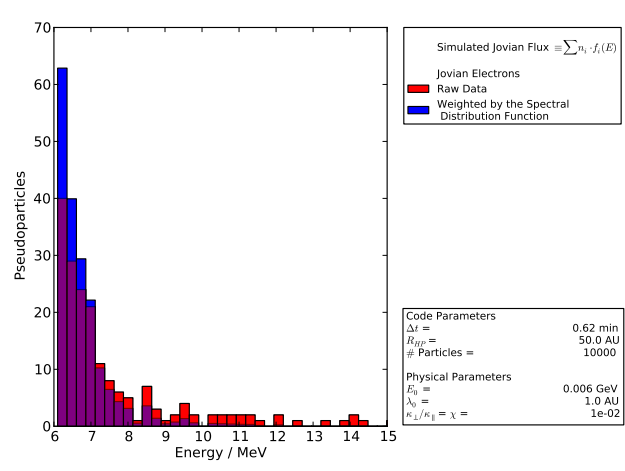

Figure 3: Histogram of the energy distribution for an injection point arbitrary well connected to the Jovian magnetosphere. In blue the percentage of trajectories in each bin is shown without weighting with the source spectrum, in red with the weighting method applied. Only trajectories of energies between 6 and $10 \mathrm{MeV}$ contribute substantially to the weighted differential intensity.

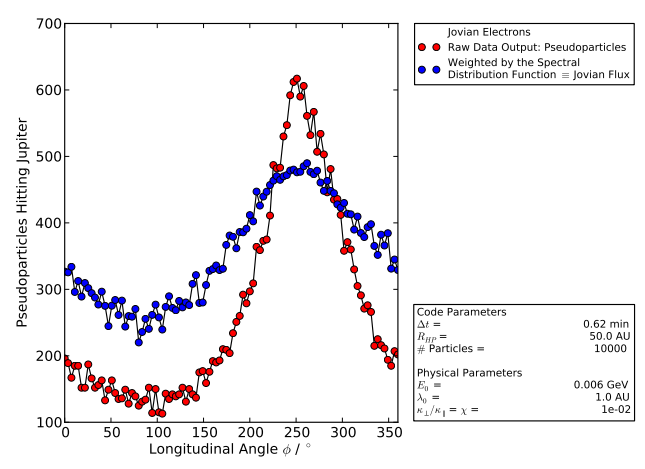

Figure 4: The trajectory counts (red) and the weighted differential intensity $j\left(E^{0}, r^{0}\right)$ over the full Earth orbit, divided into hundred single injection points. The spectral weighting smoothes out the influence of the actual trajectory counts and therefore takes the probabilities of the trajectories into account.

with the particle rigitiy $P_{0}$ at the injection point and the total amount of trajectories $N$. In Figure 3 the effect of this spectral weighting on the pseudo-particle counts is shown for the Jovian exit position, while Figure 4 shows how $j_{j o v}\left(E^{0}, r^{0}\right)$ depends on the spectrum.

If the simulated differential electron intensities are to be compared with spacecraft data, the temporal resolution of the data has to be in agreement with the propagation time of the simulated pseudo-particles, due to the changing relative position and magnetic connection of Jupiter's magnetosphere.

Similar as explained for $j\left(E^{0}, r^{0}\right)$, also the propagation times of the trajectories are weighted with the source spectra in order to obtain the correct value:

$$
\tau\left(r^{0}, E^{0}\right)=\frac{\sum_{k=1}^{N} f_{J o v}\left(E_{k}^{e x i t}\right) s_{k}^{e x i t}}{\sum_{k=1}^{N} f_{J o v}\left(E_{k}^{e x i t}\right)}
$$

Figure 6 shows, in contrast to Figure 5, the weighted propagation times in relation to the corresponding energies the pseudo-particles gained, above the injection energy of $6 \mathrm{MeV}$. Accordingly, the main part takes not more than two days to cover the distance between the Earth orbit and Jovian's magnetosphere. Related to that, without diffusive effects the pseudo-particles' mean propagation time along the Parker spiral was identified as ca. 0.7 days.

\section{Simulated Jovian Electron Intensities}

The simulation setup considers the heliopause only as an outer boundary of the parameter space, as this study focuses on electron fluxes in the inner heliosphere. Although, different radii 


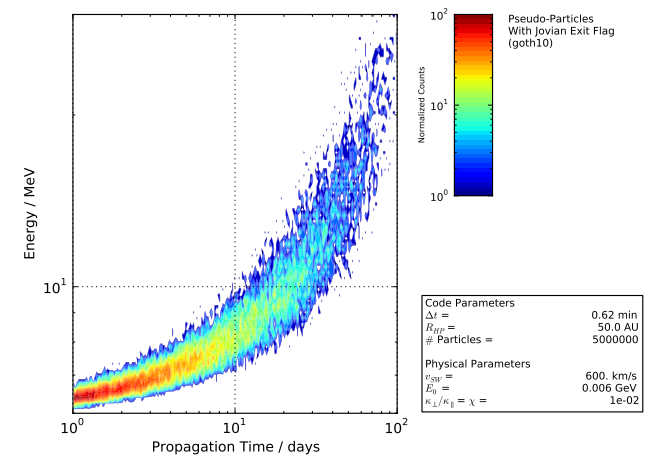

Figure 5: Histogram showing the number of trajectories in relation to their propagation time and gained exit energy. To compare the distribution rather than the actual counts, the histogram is normalized to hundred at the maximum bin.

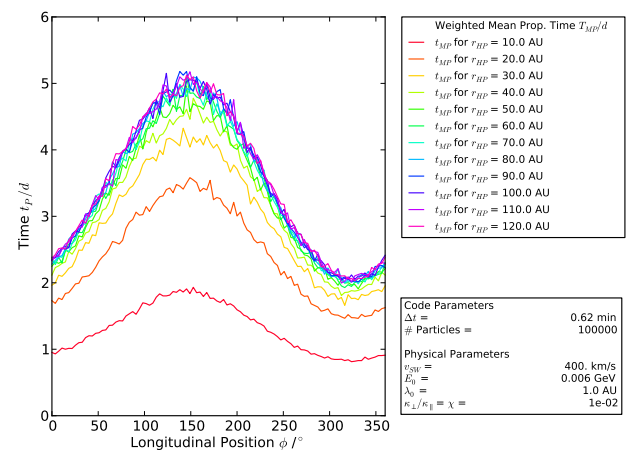

Figure 7: Mean propagation times $\tau\left(r^{0}, E^{0}\right)$ over a full Earth orbit, simulated with varying Heliospheric radii $R_{H P}$ from 10 up to $120 \mathrm{AU}$.

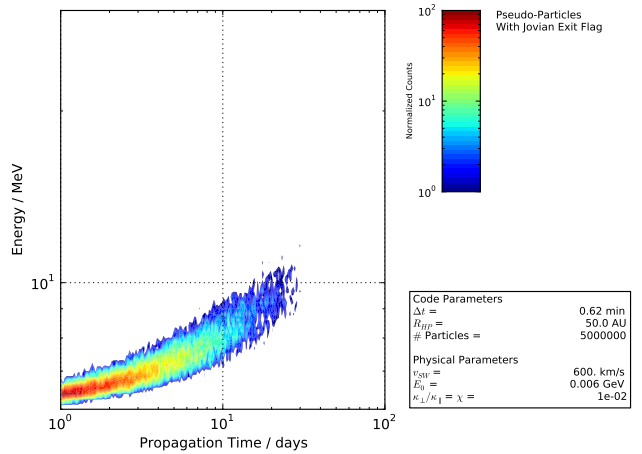

Figure 6: Similar histogram to Figure 5 but with respect to the propagation time weighted with the Jovian source spectrum $j_{J o v}\left(E^{\text {exit }}\right)$.

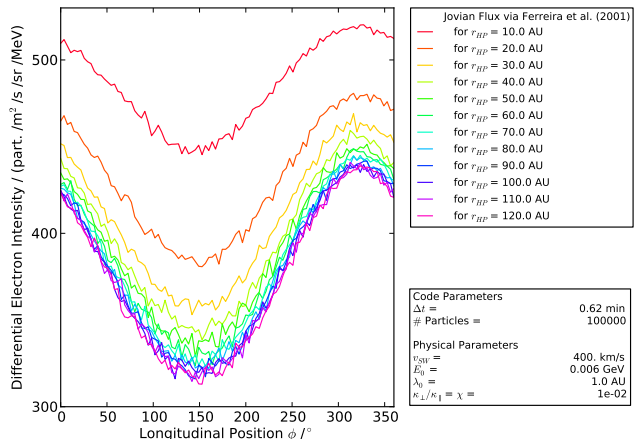

Figure 8: Differential intensities $j\left(E^{0}, r^{0}\right)$, obtained similar to $\tau\left(r^{0}, E^{0}\right)$ in Figure 7 .

$R_{H P}$ of this outer boundary were investigated to identify their influence on the simulated Jovian electron fluxes. Figures 7 and 8 show the weighted mean propagation time and the differential intensity of the simulated Jovian electrons as a function of the distance of the outer boundary $R_{H P}$. Both the mean propagation time and the Jovian intensity variation converge for $R_{H p}>50 \mathrm{AU}$, indicating that only trajectories reaching out to this extend statistically contribute to the simulated Jovian electron intensity composition.

The influence of the solar wind speed $u_{S W}$ is shown in Figure 9. The longitudinal shift of the intensity peak due to the best magnetic connection with the Jovian source is caused by the changing curvatures of the spiral Parker field lines with higher $u_{S W}$. Furthermore, the intensity variation between trajectory points of good and bad magnetic connection is smoothed out with decreasing solar wind speed because the longer field line combined with slower convection increase 


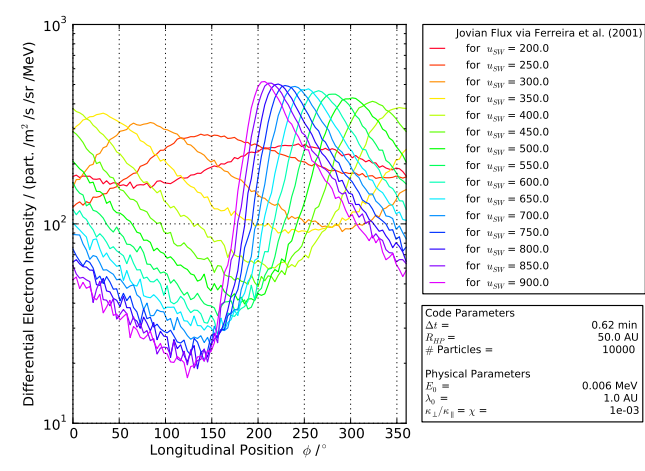

Figure 9: Differential intensities $j\left(E^{0}, r^{0}\right)$ simulated with solar wind speed values of $u_{S W}=$ 200 to $u_{S W}=900 \mathrm{~km} / \mathrm{s}$. The $\kappa_{\perp}$ to $\kappa_{\|}$ratio is assumed as $\chi=0.001$ and $\lambda_{0}=1$ AU according to Eqn. 2.5.

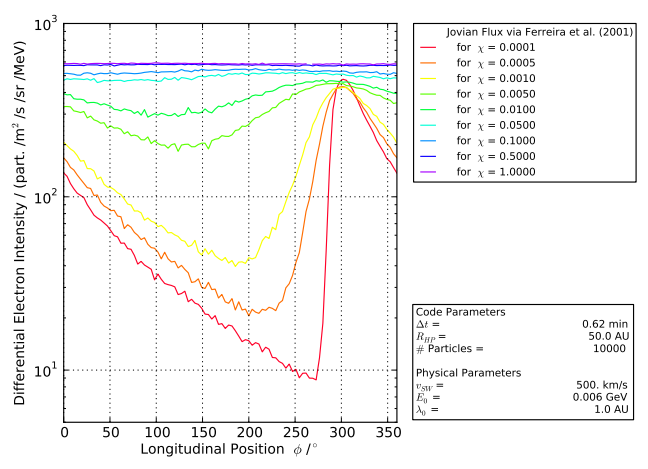

Figure 10: Differential intensities $j\left(E^{0}, r^{0}\right)$ simulated with $\kappa_{\perp}$ to $\kappa_{\|}$ratios of $\chi=0.0001$ to $\chi=1.0$. The solar wind speed is assumed as $u_{S W}=500 \mathrm{~km} / \mathrm{s}$ and $\hat{\kappa}$ is defined via $\lambda_{0}=$ $1 \mathrm{AU}$ as for Figure 9

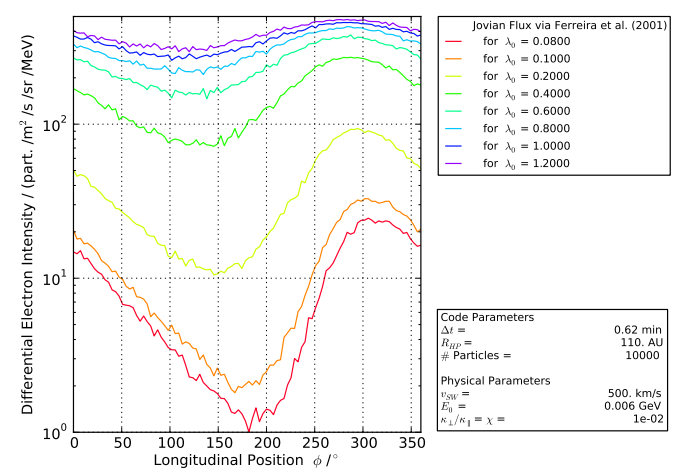

Figure 11: Differential intensities $j\left(E^{0}, r^{0}\right)$ simulated for different scaled $\hat{\kappa}$ via $\lambda_{0}=0.08 \mathrm{AU}$ to $\lambda_{0}=1.2 \mathrm{AU}$. Similar to Figure 10 the solarwindspeed is assumed as $u_{S W}=500 \mathrm{~km} / \mathrm{s}$ and $\chi=0.01$ is set as in Figure 9 .

the possibility of perpendicular diffusion.

A similar effect can be seen in Figure 10 where the ratio $\chi=\kappa_{\perp} / \kappa_{\|}$is varied. With a constant solar wind speed of $u_{S W}=500 \mathrm{~km} / \mathrm{s}$ the intensity peak does not change its longitudinal position but only its shape, and smooths out due to the increasing influence of perpendicular diffusion.

In contrast the Variation of the strength of the diffusion via the mean free path $\lambda_{0}$ as depicted in Figure 11 should not effect the shape and position but the absolute value of the intensity distribution, spreading over three orders of magnitude. The weak variation in $\phi$ can be interpreted as an effect of the backward time-step $d s$ and its influence on the diffusive processes. 


\section{Summary And Conclusion}

We presented the first results of a newly developed, GPU-accelerated SDE code to simulate Jovian electron densities in the inner heliosphere. The variation of $u_{S W}, \chi=\kappa_{\perp} / \kappa_{\|}$and $\lambda_{0}$, as shown in the Figures 9, 10 and 11, shows reliable results, comparable to preliminary studies by [Strauss et al.(2011b), Strauss et al.(2013)].

For future work our code offers the possibility of extensive parameter studies, in order to investigate the transport parameters qualitatively and quantitatively. For instance, the opportunity to simulate electron intensities for arbitrary injection points, allows to compare data of several spacecraft with parameter studies, covering the same trajectory. Furthermore, we are able to use spacecraft data as input parameters, such as the solar wind speed $u_{S W}$ measured at the particular injection point.

\section{Acknowledgements}

This work was carried out within the framework of the bilateral BMBF-NRF-project 'Astrohel' (01DG15009) funded by the Bundesministerium für Bildung und Forschung. The responsibility of the contents of this work is with the authors.

\section{References}

[Burger et al.(2008)] Burger, R. A.et al., 2008, ApJ, 674, 511-519

[Dunzlaff et al.(2015)] Dunzlaff, P. et al., 2015, Computer Physics Communications, 192, 156-165

[Ferreira et al.(2001)] Ferreira, S. E. S. et al., 2001, JGR, 106, 24979-24988

[Kopp et al.(2012)] Kopp, A et al., 2012, Computer Physics Communications, 183, 530-542

[Parker (1965)] Parker, E. N., 1965, Planetary and Space Science, 13, 9-49

[Strauss et al.(2011a)] Strauss, R. D. et al. 2011, JGR, 116, 12105

[Strauss et al.(2011b)] Strauss, R. D. et al. 2011, ApJ, 735, 83

[Strauss et al.(2013)] Strauss, R. D. et al. 2013, Advances in Space Research, 51, 339-349

[Zhang (1999)] Ming Zhang, 1999, ApJ, 513, 409 